\title{
Factores explicativos de los resultados empresariales: un estudio de las empresas españolas
}

\author{
Huerta, Patricia* \\ Contreras, Sergio** \\ Almodóvar, Paloma*** \\ Pedraja-Rejas, Liliana****
}

\section{Resumen}

Los resultados empresariales se explican por diversos factores, que a su vez, influyen en la supervivencia de la empresa. Es por ello, que el presente artículo persigue dos objetivos. En primer lugar, realizar una revisión bibliográfica de los factores que explican e influyen en los resultados empresariales, y en segundo lugar, contrastar los planteamientos teóricos para aportar evidencia empírica al respecto, a través de un análisis comparativo con estudios previos. La contrastación se realiza sobre un panel completo de empresas industriales españolas, para el periodo 2000-2005. Utilizando análisis de componentes de varianza se identifican los factores que explican el $95.6 \%$ de los resultados empresariales y empleando un modelo de efectos aleatorios se descubre que éstos también influyen en los resultados obtenidos. Los resultados del estudio sugieren que el efecto empresa es 17.8 veces superior con respecto al efecto sector en la determinación de los resultados empresariales; y el

\section{Recibido: 15-02-11. Aceptado: 04-11-11}

* Licenciada en Ciencias Administrativas. Ingeniero Comercial. Diplomada en Marketing (Universidad del Bío-Bío. Chile). Doctora en Dirección de Empresas (Universidad Complutense de Madrid. España). Profesora de la Facultad de Ciencias Empresariales. Universidad del Bío-Bío (Chile).e-mail: phuerta@ubiobio.cl.

** Licenciado en Matemática. Ingeniero Matemático. Magíster en Estadística (Universidad de Concepción). Doctor en Métodos de Apoyo a la Decisión (Pontificia Universidad Católica de Río de Janeiro). Profesor de la Facultad de Ciencias (Universidad del Bío-Bío. Chile). e-mail: scontre@ubiobio.cl

*** Licenciada en Administración y Dirección de Empresas. Master en Análisis de Datos y Construcción de Modelos. Doctora en Dirección de Empresas (Universidad Complutense de Madrid. España). Profesora de la Facultad de Ciencias Económicas y Empresariales (Universidad Complutense de Madrid. España).e-mail: paloma.almodovar@ccee.ucm.es

**** Ingeniero Comercial (Universidad de Tarapacá). Máster en Dirección y Marketing. Doctora en Administración y Dirección de Empresas (Universidad Politécnica de Valencia. España). Profesora de la Universidad de Tarapacá. e-mail: Ipedraja@uta.cl 
Factores explicativos de los resultados empresariales: un estudio de las empresas... Huerta, Patricia; Contreras, Sergio; Almodóvar, Paloma y Pedraja-Rejas, Liliana

efecto localización explica el $7.39 \%$ de los resultados. Finalmente, se concluye, que los factores sector, tiempo, localización, y principalmente el factor empresa, deberán ser considerados por aquellas organizaciones que desean alcanzar mejores resultados.

Palabras clave: Resultados empresariales, performance, empresas, España.

\title{
Explanatory Factors for Business Results: A Study of Spanish Enterprises
}

\begin{abstract}
Business results can be explained by diverse factors that, in turn, influence the survival of an enterprise. Therefore, this article pursues two objectives: first, to make a bibliographic review of the factors that explain and influence business results, and second, to contrast the theoretical proposals in order to contribute empirical evidence through a comparative analysis with earlier studies. The contrasting is carried out using a complete panel of Spanish industrial enterprises for the period 20002005. Employing variable component analysis, factors that explain $95.6 \%$ of business results are identified and, using a random effects model, it is discovered that these also influence results. Results of the study suggest that the effect of the company is 17.8 times higher in relation to the effect of the sector on determining entrepreneurial results; and the locality effect explains $7.39 \%$ of the results. Finally, conclusions are that the factors of sector, time and locality, and, principally, the factor of company ought to be considered by those organizations that wish to achieve better results.
\end{abstract}

Keywords: Business results, performance, enterprises, Spain.

\section{Introducción}

Diversos estudios se han generado en el área de dirección estratégica y economía industrial, los cuales buscan identificar factores que provocan que algunas organizaciones obtengan más éxito que otras (Rubio y Aragón, 2002; Aragón y Rubio, 2005; Iglesias et al., 2007), o bien, que sean más rentables (Claver et al., 2002; Capar, 2009; Huang y Sylvie, 2010). En esta línea la literatura de dirección estratégica se ha planteado interrogantes, siendo una de las más recurrentes el por qué algunas empresas obtienen distintos niveles de rentabilidad (Barney, 1991; Grant, 1991; Amit y Schoemaker,
1993; Peteraf, 1993; Wernerfelt, 1984). A pesar de la amplia discusión sobre el tema aún no existe consenso generalizado sobre cuáles son los factores que realmente influyen en los resultados de las empresas (Iglesias et al., 2007) tradicionalmente operacionalizados a través de medidas cuantitativas y cualitativas (Calderón et al., 2010: 15) y por ello el debate no ha terminado (Halkos y Tzeremen, 2007: 714) y sigue sin resolverse (Chen, 2009: 298) generando permanentes trabajos, tales como los de Ariño, Ariño y Garcia-Castro (2008); Chen (2009) y Huang y Sylvie (2010), entre otros. Sin embargo, pese a que la mayoría de los estudios analizan principalmente si es el 
efecto empresa o el efecto industria el que mejor explica la varianza de los resultados obtenidos (Chen, 2009: 297), ellos no han recibido mucha atención empírica debido a la dificultad en hacer operativo el constructo teórico de las características estructurales de una industria y las características de las empresas (Huang y Silvie, 2010: 4).

Además, debido a que las conclusiones alcanzadas en los estudios pasados plantean que sus resultados son específicos a las empresas de una zona geográfica y de un período de tiempo muy concreto (Iglesias et al., 2007), permiten destacar la importancia de analizar el efecto país como determinante de los resultados empresariales (Rubio y Aragón, 2002: 52; Chen, 2009). Por esta razón, este artículo genera un aporte al conocimiento, actualizando el periodo aplicado en estudios pasados e incorporando otro factor relevante pero excluido de la mayoría de las investigaciones tradicionales, como es la localización geográfica. A la vez, con la finalidad de realizar un análisis comparativo se estudiarán las empresas industriales, debido a que la mayoría de las investigaciones emplea esta categoría (Schmalensee, 1985; Rumelt, 1991; McGahan y Porter, 1997; Galán y Vecino, 1997; Roquebert et al., 1996; Mauri y Michaels, 1998; González, 2000).

Por lo anterior, con la finalidad de contribuir en evidenciar los factores que explican e influyen en los resultados que alcanzan las organizaciones, se trabaja en función de dos objetivos. En primer lugar, realizar una revisión bibliográfica sobre el tema, y en segundo lugar, contrastar los planteamientos anteriores para aportar evidencia empírica al respecto.
Específicamente, este trabajo avanza en la discusión del tema, incorporando en un solo artículo ambos aspectos, explicación e influencia de los factores. Aspectos que en la mayoría de las investigaciones se analizan por separado, a pesar de su estrecha vinculación.

En particular, se construye un panel completo y balanceado de empresas, para el periodo $2000-2005$, seis años, obteniendo una muestra final de $1.157 \mathrm{em}$ presas y 6.942 observaciones. El error muestral fue del 0.026 a un nivel de confianza del 95\%. Específicamente, en esta investigación se emplea la base de datos de la Encuesta Sobre Estrategias Empresariales (ESEE, 1990) que reúne información de 20 sectores industriales españoles y es generada por la Sociedad Estatal de Participaciones Estatales (SEPI). Se opta por utilizar base de datos porque permite obtener información longitudinal, la cual es usualmente utilizada en el área de economía y dirección (Huang y Sylvie, 2010: 17).

Para la medición de las variables del estudio se mantuvo como criterio que hayan sido utilizadas en estudios similares. Específicamente, en el caso de la variable dependiente, resultados empresariales $(\mathrm{RE})$, se midió a través del logaritmo natural del valor añadido anual de la empresa (en euros); el efecto sector (ES) se midió a través del sector industrial principal en el cual se encuentra la empresa; el efecto empresa (EE) fue medido a través de número que identifica la empresa; el efecto localización (EL) se midió a través de la ubicación geográfica de la empresa en el país; el efecto tiempo (ET) fue medido a través del año de la observación; la variable control tamaño $(T)$, fue medida a través 
Factores explicativos de los resultados empresariales: un estudio de las empresas... Huerta, Patricia; Contreras, Sergio; Almodóvar, Paloma y Pedraja-Rejas, Liliana

del logaritmo natural del número anual de empleados que posee la empresa. Finalmente, para la contrastación de las hipótesis se emplearon correlaciones, análisis de componentes de varianza y técnicas para datos de panel, a través de un modelo de efectos aleatorios.

\section{Resultados empresariales}

A lo largo de los estudios, se observan diferentes formas de medir los resultados empresariales, pero a pesar de la forma de medida utilizada para determinar los resultados o performance siguen concentrando el interés de los investigadores (Huerta et al., 2010). De hecho, tras la revisión de la literatura se descubrió que una de las formas de medida más utilizadas en los estudios de dirección estratégica y economía industrial, es el ROA (Cool et al., 1988; Short et al., 2007; Hull y Rothenberg, 2008; Tanriverdi y Lee 2008; Wiersema y Bowen, 2008), la $Q$ de Tobin (Wernerfelt y Montgomery, 1988; Lang y Stulz, 1994; Ramírez y Espitia, 2002; MiIler, 2006), las ventas (Benavente, 2008), el margen bruto (Merino y Rodríguez, 1997), la eficacia organizacional (Pedraja-Rejas et al., 2009) y el valor agregado (Galve y Salas, 1993; Díaz y Sanchez, 2008; Torrents, 2008). Siendo menor el número de investigaciones que emplea el valor agregado (VA) como medida de resultados, a pesar del mayor acceso a esta variable, producto de la menor cantidad de ítem que utiliza en su construcción, a diferencia de otras formas de medida como el ROA, ROI, entre otros, cuya construcción involucra más elementos. Por ello, se espera que este artículo sea un aporte en este aspecto.
En concreto, Galve y Salas (1993) plantean que el valor agregado es la mejor variable que mide el impacto de las variaciones de eficiencia interna debido a la estructura de propiedad de la empresa sobre la rentabilidad de la misma, dado que incluye toda la riqueza generada por la empresa en el ejercicio económico y se encuentra ajustado por el grado de integración vertical de la misma.

\section{Factores que explican los resultados empresariales}

Tras la revisión de la literatura se observa que en la obtención de la rentabilidad participan una serie de factores explicativos que pueden clasificarse en tres categorías (Fernández, 1992; Cuervo, 1993; Galán y Vecino, 1997): Los factores macroeconómicos, los cuales se relacionan con el marco económico general; los factores sectoriales, que se refiere a la actividad económica en la cual se encuentra inserta la empresa y; los factores empresariales que se refiere a los recursos y capacidades que poseen las organizaciones y que generan heterogeneidad en las empresas, y por ende, en sus resultados.

En general, un gran número de estudios en el área de estrategia se han enfocado en determinar las causas que explican el resultado de las empresas y algunos han considerado al ambiente macroeconómico como uno de los factores de gran influencia, a través de la interacción, directa o indirecta, con el efecto industria (Ariño et al., 2008: 629). En esta línea, Makino et al. (2004) y Peng (2001) resaltan la importancia de las diferencias económicas, políticas, sociales, culturales e institucionales que existen a lo largo de los países (Chen, 2009: 298). 
Sin embargo, en la literatura se aprecia que es menor el número de investigaciones que defiende el efecto industria o sector sobre el efecto empresa. Lo que amerita realizar una breve revisión de los principales hallazgos y características de los estudios empíricos que explican los resultados empresariales. Siendo las investigaciones más trascendentes el estudio de Schmalensee (1985) y el estudio de Rumelt (1991).

Específicamente, Schmalensee (1985) fue el primero en estimar los componentes de la varianza de los resultados (Ariño et al., 2008) y obtiene que el efecto de mayor importancia es el sectorial, resultado similar al encontrado por Wernerfelt y Montgomery (1988), Montgomery y Wernerfelt (1991) y Dunne y Macpherson (1991). En su modelo incluye tres factores que pueden influir en la rentabilidad: la industria a la que pertenece la empresa, la corporación de la que forma parte la empresa y su cuota de mercado.

Así, Schmalensee (1985) comprobó que el efecto corporación no existe y que los otros dos efectos sí son significativos, y por ello eliminó del modelo el efecto corporación, aplicando al modelo resultante el análisis de componentes de varianza. Además, obtiene que el efecto cuota de mercado era menos del $1 \%$ de la variabilidad de la rentabilidad, mientras que el efecto sector permite explicar el $19.5 \%$, quedando asignado el $80 \%$ restante al error. Sin embargo, este trabajo ha sido criticado debido a que utilizó datos de rentabilidad de un solo año (1975), y a menos que se utilice más de un dato para cada empresa (utilizando varios años), el efecto empresa irá unido al error y no podrá ser detectado (Rumelt, 1991: 171; Galán y Vecino, 1997: 23; McGahan y Porter, 1997: 17; Claver et al., 2002: 40-41).
Específicamente, se plantea que dentro del $80 \%$ de la varianza que quedaba sin explicar por el modelo, podría ir incluido el efecto de los factores internos de cada empresa, el cual no podía ser determinado con exactitud (Claver et al., 2000; Claver et al., 2002; Iglesias et al., 2007). Sin embargo, tal fue su relevancia que sigue siendo citado y utilizado de ejemplo en diversas investigaciones.

De hecho, el estudio de Schmalensee (1985) provocó un gran interés de los investigadores que a partir de ese año en adelante siguen realizando estudios sobre el tema. Por ello, tras su investigación surgieron muchos otros estudios, como los estudios de Odagiri (1983), Scott y Pascoe (1986), Cubbin y Geroski (1987) y Hansen y Wernerfelt (1989), que pese a que entregaron claras señales a los investigadores afirmando que las características internas de la empresa poseían un mayor poder explicativo en relación al sector industrial, es el estudio de Rumelt (1991) el que es recordado con mayor frecuencia.

Lo anterior se debe a que la investigación de Rumelt (1991) se enfocó en solucionar los problemas del estudio de Schmalensee (1985), ampliando los datos utilizados por él, lo que le permitió estimar el efecto unidad de negocio, descomponer el efecto sector total en un componente estable y otro coyuntural, determinando este último a través de la interacción sector-año y supera la ambigüedad terminológica, denominando efecto unidad de negocio a aquél referido a las habilidades y recursos internos de cada unidad y distinguiéndolo del efecto corporación, relativo a la pertenencia de cada unidad a una determinada corporación empresarial. 
Factores explicativos de los resultados empresariales: un estudio de las empresas... Huerta, Patricia; Contreras, Sergio; Almodóvar, Paloma y Pedraja-Rejas, Liliana

Posteriores estudios siguen la línea desarrollada por Rumelt (1991) y encuentran que es el efecto empresa el que explica un mayor porcentaje de la varianza explicada de la rentabilidad empresarial, tales como los estudios de Amel y Froeb (1991), Fernández et al. (1996, 1997), Hill y Deeds (1996), Roquebert et al. (1996), Galán y Vecino (1997), McGahan y Porter (1997), Mauri y Michaels (1998), Brush et al. (1999), McGahan (1999), Chang y Singh (2000), González (2000), Claver et al. (2002), Caloghirou et al. (2004), Iglesias et al. (2007). En definitiva, estos estudios llegan a resultados similares aunque analizan diferentes periodo de tiempo, entornos económicos heterogéneos e incluyen diversos factores al modelo.

Al contrario, dentro de ellos, el estudio de McGahan y Porter (1997) encuentra que los resultados empresariales dependen del sector. En concreto, en la industria manufacturera predominó el efecto empresa, mientras que en el resto de las industrias (tales como, transportes, servicios, entre otros) predominó el efecto sector. De manera similar, Caloghirou et al. (2004) analizando un conjunto de empresas manufactureras griegas descubren un resultado similar. Al igual que el reciente estudio de Huang y Sylvie (2010), realizado a través de una encuesta, una base de datos y aplicando regresión jerárquica, encuentran que el efecto industria posee una importancia relativa mayor sobre el efecto empresa, a lo largo de las tres medidas de resultados empleada en su investigación (crecimiento de los ingresos, rentabilidad y la relación de los resultados).

En resumen, no existe consenso sobre la relevancia de un efecto sobre otro, sólo se rescata la importancia de con- siderar el efecto empresa y el efecto sector. Sin embargo, estos estudios entregan indicios sobre la necesidad de incorporar otros factores a los modelos (Rumelt, 1991; Roqueber et al., 1996; Galán y Vecino, 1997; Claver et al., 2002), que permitan explicar mejor el fenómeno analizado, tales como el factor macroeconómico (factor tiempo) analizado preliminarmente por Rumelt (1991) y el factor localización estudiado por Iglesias et al. (2007).

\section{Modelo explicativo de los resultados empresariales: empresas industriales españolas}

El modelo empleado en este trabajo, sobre el cual se aplica el análisis de componentes de varianza, con la finalidad de descubrir la importancia relativa del efecto empresa, del efecto sector, del efecto localización y del efecto tiempo, sobre la determinación de los resultados empresariales, se observa en la siguiente fórmula.

Fórmula (1): Resultados Empresariales

$R E_{i j t}=\mu+E S E_{i}+E E_{j}+E T_{t}+E L_{j}+\varepsilon_{i j t}$

De esta forma, este modelo sigue a Schmalensee (1985), Rumelt (1991), Chen (2009) y Claver et al. (2002), entre otros, y plantea que $\mathrm{RE}_{\mathrm{ijt}}$, es decir, los resultados en el año $t$ (con $t=2000, \ldots, 2005)$ de la empresa $j$ (con $j=1, \ldots . n$ ) perteneciente a la industria i (con i = 1(Ind. Cárnica, ...,n) viene explicada por la constante u del modelo, y además por el ES que se refiere al efecto sector estable, es decir, a todos los impactos persistentes del sector sobre los resultados de la empresa. La existencia de este efecto indicaría que existen factores industriales (barreras de entrada, ratios de creci- 
miento, rivalidad competitiva) que se traducen en diferencias de rentabilidad entre las empresas pertenecientes a diferentes sectores; $\mathrm{EE}_{\mathrm{j}}$ que representa el efecto empresa y, en concreto, las diferencias persistentes entre empresas que son debidas a la presencia de recursos específicos de cada una de ellas; $\mathrm{ET}_{\mathrm{t}}$ reflejará el efecto del tiempo o de los años, es decir, es el efecto del sector general o macroeconómico, que reflejará las fluctuaciones anuales en variables económicas que afectan por igual a todas las unidades de negocio, como tipo de cambio, ciclo económico, inflación, entre otros;el $\mathrm{EL}_{\mathrm{j}}$ que reflejará el efecto localización o ubicación geográfica de la empresa y el $\varepsilon_{\mathrm{ijt}}$ que se referirá al error, y que puede considerarse, como señala Rumelt (1991: 173), las variaciones anuales en los resultados que son específicas de cada una de las empresas.

En resumen, este estudio pretende aportar empíricamente a la explicación de los resultados de las empresas, tema que sigue concentrando el interés de los investigadores, y que todavía no está explicado completamente (Claver et al., 2002: 49), tal como lo demuestran los recientes estudios de Serrasqueiro y Macas (2008); Garengo (2009); Kim (2009); Paliwal (2010), Huerta et al. (2010), entre otros. Lo que permite afirmar que todavía se puede contribuir en su comprensión y debate y más aún en su ámbito de aplicación, como son las empresas industriales españolas.

Dada la argumentación anterior se presentan las siguientes hipótesis de investigación:

Hipótesis 1: Los resultados empresariales de las empresas industriales españolas se explican por el factor tiempo, el factor sectorial, el factor de localización y el factor empresarial, siendo este último el que mayor explicación aporta al modelo.

Hipótesis 2: El factor tiempo, el factor sectorial, el factor de localización y el factor empresarial influyen en los resultados empresariales de las empresas españolas.

Cuya confirmación o comprobación se constatará a través de los párrafos a continuación:

Específicamente, la Tabla 1 presenta los estadísticos descriptivos generales, se destaca la media de los resulta-

\section{Tabla 1}

\section{Estadísticos descriptivos}

\begin{tabular}{lccccc}
\hline Variables & N & Mínimo & Máximo & Media & Desv. Típica \\
\hline Efecto Empresa & 6.942 & 1 & 3.430 & 1.931 .56 & 1.036 .699 \\
Efecto Localización & 6.942 & 1 & 17 & 9.74 & 4.057 \\
Efecto Tiempo & 6.942 & 2000 & 2005 & 2002.50 & 1708 \\
Efecto Sector & 6.942 & 1 & 20 & 10.23 & 5.293 \\
Resultados Empresariales & 6.942 & 9.09 & 20.56 & $\mathbf{1 4 . 8 2 3 6}$ & 1.89162 \\
Variable control: & & & & & \\
Tamaño & 6.942 & 0.69 & 9.47 & 4.3203 & 1.50222 \\
Trabajadores & 6.942 & 2 & 12939 & 267.76 & 813.939 \\
N válido (según lista) & 6.942 & & & & \\
\hline
\end{tabular}

Fuente: SPSS Versión 19. 
Factores explicativos de los resultados empresariales: un estudio de las empresas... Huerta, Patricia; Contreras, Sergio; Almodóvar, Paloma y Pedraja-Rejas, Liliana

dos empresariales de las empresas industriales españolas, con un valor de $14.8 \%$, con un promedio de 267 trabajadores, considerando un máximo de 20 sectores y 17 localidades en las cuales se ubican las organizaciones analizadas.
La Tabla 2 presenta la distribución del tamaño y los resultados empresariales por sector industrial y localización. Esta tabla permite apreciar que existe una mayor concentración de mano de obra promedio en el sector de vehículos de motor y en la

Tabla 2

Distribución del tamaño y los resultados empresariales por sector industrial y localización

\begin{tabular}{|c|c|c|c|c|c|c|}
\hline $\mathbf{N}^{\circ}$ & Sector & $\begin{array}{c}\text { Media } \\
\text { Trabajadores }\end{array}$ & $\begin{array}{c}\text { Media } \\
\text { Resultados }\end{array}$ & Localización & $\begin{array}{c}\text { Media } \\
\text { Trabajadores }\end{array}$ & $\begin{array}{c}\text { Media } \\
\text { Resultados }\end{array}$ \\
\hline 1 & Ind. Cárnica & 232.91 & 14.83 & 1 Andalucía & 191.91 & 14.28 \\
\hline 2 & Prod. Alim. y Tabaco & 210 & 14.40 & 2 Aragón & 413.94 & 14.96 \\
\hline 3 & Bebidas & 452.48 & 16.54 & 3 Asturias & 615.58 & 15.83 \\
\hline 4 & Textil & 104.29 & 13.78 & 4 Baleares & 30.50 & 13.20 \\
\hline 5 & Cuero y Calzado & 42.19 & 13.40 & 5 Canarias & 154.89 & 15.08 \\
\hline 6 & Ind. De la Madera & 123.76 & 14.02 & 6 Cantabria & 242.23 & 15.76 \\
\hline 7 & Papel & 243.19 & 15.26 & $\begin{array}{l}7 \text { Castilla- } \\
\text { la Mancha }\end{array}$ & 144.17 & 13.96 \\
\hline 8 & $\begin{array}{l}\text { Edición y Artes } \\
\text { Graficas }\end{array}$ & 204.91 & 14.70 & 8 Castilla León & 520.76 & 15.14 \\
\hline 9 & Prod. Químicos & 304.86 & 15.88 & 9 Cataluña & 263.33 & 15.17 \\
\hline 10 & $\begin{array}{l}\text { Prod. de Caucho y } \\
\text { Plástico }\end{array}$ & 313.97 & 14.90 & $\begin{array}{l}10 \text { Comunidad } \\
\text { Valenciana }\end{array}$ & 207.88 & 14.21 \\
\hline 11 & $\begin{array}{l}\text { Prod. de Minerales no } \\
\text { metálicos }\end{array}$ & 264.84 & 14.94 & 11 Extremadura & 120.48 & 13.78 \\
\hline 12 & Metalúrgica & 509.28 & 16.18 & 12 Galicia & 421.86 & 14.95 \\
\hline 13 & Prod. Metálicos & 133.41 & 14.57 & 13 Madrid & 162.89 & 14.65 \\
\hline 14 & $\begin{array}{l}\text { Maquinas y Equipos } \\
\text { Mecánicos }\end{array}$ & 233.66 & 14.89 & 14 Murcia & 317.69 & 14.99 \\
\hline 15 & $\begin{array}{l}\text { Maq. de Ofc.. Eq. } \\
\text { Informaticos }\end{array}$ & 183.92 & 14.67 & 15 Navarra & 488.11 & 15.60 \\
\hline 16 & $\begin{array}{l}\text { Maq.. Materiales } \\
\text { elect. Electrónicos }\end{array}$ & 239.35 & 15.02 & 16 País Vasco & 369.32 & 15.69 \\
\hline 17 & Vehículos de Motor & 1175.77 & 16.27 & 17 La Rioja & 72.30 & 14.06 \\
\hline 18 & $\begin{array}{l}\text { Otro material de } \\
\text { transporte }\end{array}$ & 194.97 & 15.32 & Promedio & 278.70 & 14.8 \\
\hline 19 & Muebles & 123.39 & 13.88 & & & \\
\hline 20 & $\begin{array}{l}\text { Otras Ind. } \\
\text { Manufactureras }\end{array}$ & 129.48 & 14.28 & & & \\
\hline Promedio & & 271.03 & 14.8 & & & \\
\hline
\end{tabular}

Fuente: Elaboración propia. 
Tabla 3. Correlaciones

\begin{tabular}{|c|c|c|c|c|c|c|c|c|}
\hline \multicolumn{2}{|c|}{ Variables } & \multirow{2}{*}{$\begin{array}{c}\text { RE } \\
1\end{array}$} & \multirow[t]{2}{*}{ EE } & \multirow[t]{2}{*}{ ES } & \multirow[t]{2}{*}{ EL } & \multirow[t]{2}{*}{ ET } & \multirow[t]{2}{*}{ Tamaño } & \multirow[t]{2}{*}{ Trabaj } \\
\hline RE & $\begin{array}{l}\text { Correlación } \\
\text { de Pearson }\end{array}$ & & & & & & & \\
\hline & Sig. (bilateral) & & & & & & & \\
\hline & $\mathrm{N}$ & 6942 & & & & & & \\
\hline \multirow[t]{3}{*}{ EE } & $\begin{array}{l}\text { Correlación } \\
\text { de Pearson }\end{array}$ & -0.015 & 1 & & & & & \\
\hline & Sig. (bilateral) & $\underline{0.202}$ & & & & & & \\
\hline & $\mathrm{N}$ & 6942 & 6942 & & & & & \\
\hline \multirow[t]{3}{*}{ ES } & $\begin{array}{l}\text { Correlación } \\
\text { de Pearson }\end{array}$ & $\begin{array}{c}0.103 \\
\left({ }^{* *}\right)\end{array}$ & $\begin{array}{c}0.035 \\
\left({ }^{* *}\right)\end{array}$ & 1 & & & & \\
\hline & Sig. (bilateral) & $\underline{0.000}$ & 0.003 & & & & & \\
\hline & $\mathrm{N}$ & 6942 & 6942 & 6942 & & & & \\
\hline \multirow[t]{3}{*}{ EL } & $\begin{array}{l}\text { Correlación } \\
\text { de Pearson }\end{array}$ & $\begin{array}{c}0.063 \\
\left({ }^{* *}\right)\end{array}$ & 0.023 & $\begin{array}{c}0.112 \\
\left({ }^{* *}\right)\end{array}$ & 1 & & & \\
\hline & Sig. (bilateral) & $\underline{0.000}$ & 0.055 & 0.000 & & & & \\
\hline & $\mathrm{N}$ & 6942 & 6942 & 6942 & 6942 & & & \\
\hline \multirow[t]{3}{*}{ ET } & $\begin{array}{l}\text { Correlación } \\
\text { de Pearson }\end{array}$ & 0.023 & 0.000 & -0.001 & 0.000 & 1 & & \\
\hline & Sig. (bilateral) & $\underline{0.057}$ & 1.000 & 0.927 & 0.997 & & & \\
\hline & $\mathrm{N}$ & 6942 & 6942 & 6942 & 6942 & 6942 & & \\
\hline \multirow[t]{3}{*}{ Tamaño } & $\begin{array}{l}\text { Correlación } \\
\text { de Pearson }\end{array}$ & $\begin{array}{c}0.949 \\
\left({ }^{* *}\right)\end{array}$ & 0.012 & $\begin{array}{c}0.092 \\
\left({ }^{* *}\right)\end{array}$ & $\begin{array}{c}0.038 \\
\left({ }^{* *}\right)\end{array}$ & -0.001 & 1 & \\
\hline & Sig. (bilateral) & $\underline{0.000}$ & 0.317 & 0.000 & 0.001 & 0.907 & & \\
\hline & $\mathrm{N}$ & 6942 & 6942 & 6942 & 6942 & 6942 & 6942 & 1 \\
\hline \multirow[t]{3}{*}{ Trabaj. } & $\begin{array}{l}\text { Correlación } \\
\text { de Pearson }\end{array}$ & $\begin{array}{c}0.518 \\
\left({ }^{* *}\right)\end{array}$ & $\begin{array}{c}0.031 \\
\left({ }^{* *}\right)\end{array}$ & $\begin{array}{c}0.082 \\
\left({ }^{* *}\right)\end{array}$ & -0.008 & 0.002 & $\begin{array}{c}0.564 \\
\left({ }^{* *}\right)\end{array}$ & \\
\hline & Sig. (bilateral) & $\underline{0.000}$ & 0.009 & 0.000 & 0.482 & 0.857 & 0.000 & \\
\hline & $\mathrm{N}$ & 6942 & 6942 & 6942 & 6942 & 6942 & 6942 & 6942 \\
\hline
\end{tabular}

Fuente: SPSS. Versión 19.

localización de Asturias. Y a su vez, existe una menor concentración de mano de obra promedio en el sector de cuero y calzado en Baleares. A su vez, el sector de bebidas y el sector de cuero y calzado, poseen una media de resultados superior en relación al resto de las industrias, al igual que la comunidad Asturias y Baleares.

Por otra parte, la Tabla 3 presenta las correlaciones del estudio. Se destaca la relación positiva y significativa entre los resultados empresariales con el efecto sector $(E S=p=0,000)$, el efecto localización $(E L=$ 
Tabla 4

Modelo de componentes de varianza de los resultados empresariales

\begin{tabular}{cccc}
\hline Efectos & Estimación & Porcentaje & Porcentaje acumulado \\
\hline Empresa & 2.902 & $83.46 \%$ & 88.46 \\
Sector & 0.163 & $4.69 \%$ & 88.15 \\
Tiempo & 0.002 & $0.05 \%$ & 88.2 \\
Localización & 0.257 & $7.39 \%$ & 95.6 \\
Error & 0.153 & $4.40 \%$ & 100 \\
Total & 3.477 & 100 & \\
\hline
\end{tabular}

Fuente: SPSS. Versión 19.

Tabla 5

Análisis comparativo con estudios previos (porcentajes)

\begin{tabular}{lcccccccc}
\hline Efectos & $\begin{array}{c}\text { Rumelt } \\
(\mathbf{1 9 9 1 )})^{\mathbf{a}}\end{array}$ & $\begin{array}{c}\text { Roqueber, } \\
\text { et al. (1996) }\end{array}$ & $\begin{array}{c}\text { Galán y } \\
\text { Vecino } \\
(\mathbf{1 9 9 7 )}\end{array}$ & $\begin{array}{c}\text { McGahan } \\
\text { y Porter } \\
(\mathbf{1 9 9 7 )}\end{array}$ & $\begin{array}{c}\text { Mauri y } \\
\text { Michaels } \\
(\mathbf{1 9 9 8 )}\end{array}$ & $\begin{array}{c}\text { González Claver et } \\
(\mathbf{2 0 0 0 )}\end{array}$ & $\begin{array}{c}\text { Estudio } \\
\text { al. (2002) }\end{array}$ & \\
\hline Tiempo & 0.05 & 0.5 & 2.41 & 2.34 & $\mathrm{~N} / \mathrm{E}$ & 4.8 & 0.36 & 0.05 \\
Sector & 4.04 & 10.2 & 1.92 & 10.81 & 6.2 & 13.2 & 2.06 & 4.68 \\
Empresa & 44.16 & 37.1 & 32.83 & 35.45 & 36.9 & 31.2 & 42.69 & $\mathbf{8 3 . 4 6}$ \\
Tiempo-Sector & 5.33 & 2.3 & 0.93 & $\mathrm{~N} / \mathrm{E}$ & $\mathrm{N} / \mathrm{E}$ & $\mathrm{N} / \mathrm{E}$ & 2.78 & $\mathrm{~N} / \mathrm{E}$ \\
Corporación & 1.64 & 17.9 & $\mathrm{~N} / \mathrm{E}$ & 0 & $\mathrm{~N} / \mathrm{E}$ & $\mathrm{N} / \mathrm{E}$ & $\mathrm{N} / \mathrm{E}$ & $\mathrm{N} / \mathrm{E}$ \\
Grupo & $\mathrm{N} / \mathrm{E}$ & $\mathrm{N} / \mathrm{E}$ & $\mathrm{N} / \mathrm{E}$ & $\mathrm{N} / \mathrm{E}$ & $\mathrm{N} / \mathrm{E}$ & 14.3 & $\mathrm{~N} / \mathrm{E}$ & $\mathrm{N} / \mathrm{E}$ \\
Localización & $\mathrm{N} / \mathrm{E}$ & $\mathrm{N} / \mathrm{E}$ & $\mathrm{N} / \mathrm{E}$ & $\mathrm{N} / \mathrm{E}$ & $\mathrm{N} / \mathrm{E}$ & $\mathrm{N} / \mathrm{E}$ & $\mathrm{N} / \mathrm{E}$ & 7.39 \\
Error & 44.78 & 32 & 61.91 & 53.67 & 56.9 & 36.5 & 52.11 & 4.40 \\
Total & 100 & 100 & 100 & $102.27^{\mathbf{b}}$ & 100 & 100 & 100 & 100 \\
\hline
\end{tabular}

N/E: No estimado;

a: Resultados obtenidos en su muestra B.

Fuente: Adaptado de Claver et al. (2002).

$p=0,000$ ), la variable control tamaño $(p=0,000)$ y en menor medida el efecto tiempo $(E T=p=0,057)$. Sin embargo, el efecto empresa presentó una relación negativa y no significativa $(E E=p=0,202)$ con los resultados empresariales.

La Tabla 4 muestra los resultados del análisis de componentes de la varianza de los resultados empresariales de las empresas industriales. Como se puede observar, el efecto empresa es superior al resto de los efectos, en línea con los resultados obtenidos en los estudios pasados. Espe- cíficamente, el efecto empresa es 17.8 veces superior con respecto al efecto sector, en la determinación de los resultados empresariales. La tabla también permite apreciar la relevancia de incorporar en el modelo un efecto que ha sido utilizado en menor medida en los trabajos anteriores, el efecto localización, el cual explica el $7.39 \%$ de los resultados empresariales.

Se destaca el menor error alcanzado con el modelo propuesto, quedando sólo un $4.4 \%$ asociado a variables que no 
se encuentran recogidas en él. Resultado no alcanzado en estudios anteriores, tal como se aprecia en la Tabla 5.

Los hallazgos permiten confirmar la hipótesis 1 , es decir, los resultados empresariales de las empresas industriales españolas se explican por el factor tiempo, el factor sectorial, el factor de localización y el factor empresarial, siendo este último el que mayor explicación aporta al modelo. En particular, para este trabajo se accede a datos de panel no equilibrados o paneles no balanceados, pero se crean y utilizan paneles balanceados y completos. En este estudio, se probaron los datos para diversas técnicas de datos de panel y, en concreto, el panel completo de empresas españolas se ajustó mejor a un modelo de efectos aleatorios. Además, en esta etapa se ha introducido como variable de control el tamaño empresarial, por ser una variable tradicionalmente utilizada en este tipo de estudios (Huerta et al., 2010). Y se ha desagregado cada sector y localización, como variable categórica, dejando como referencia el primer sector y la primera localización, respectivamente. Específicamente, el modelo de efectos aleatorios asume que las $k$ muestras son aleatorias de $k$ situaciones distintas y aleatorias. De modo que un valor aislado $Y_{i j}$ se puede escribir como:

Fórmula (2): Modelo de efectos aleatorios

$Y_{i j}=\mu+\mathrm{A}_{\imath}+\varepsilon_{i j} i=1, \ldots k$ y $j=1, \ldots n$

donde $\mu$ es la media global, $\varepsilon_{i j}$ son variables (una para cada muestra) distribuidas normalmente, con media 0 y varianza $\delta^{2}$ (como en el modelo I) y $A_{i}$ es una variable distribuida normalmente, independiente de las $\varepsilon_{i j}$, con media 0 y varianza $\sigma_{a}^{2}$.

En la Tabla 6 no se visualiza el efecto año y el efecto empresa, porque estos se encuentran recogidos en la programación del modelo, a través de la técnica estadística utilizada. Los hallazgos del estudio permiten apreciar que existen diferencias significativas sobre los resultados empresariales dependiendo del sector industrial o localización en la cual se encuentra la empresa. Además, como se puede observar la variable control del modelo, el tamaño, presenta una influencia positiva y significativa $(p=0.000)$ sobre los resultados de las empresas (ver Tabla 3). En definitiva, los resultados anteriores permiten confirmar la hipótesis 2 , es decir, el factor tiempo, el factor sectorial, el factor localización y el factor empresarial influyen en los resultados empresariales de las empresas industriales españolas. Por último, se observa que los estadísticos que muestran la bondad de ajuste del modelo son significativos, a través de un estadístico $\mathrm{F}$ de $565.607^{* * *} \mathrm{y}$ un $\mathrm{R}^{2}$ del $74.6 \%$.

En concreto, los resultados permiten apreciar las diferencias a lo largo de las comunidades autónomas, lo que puede explicarse por el diferente marco legislativo y la existencia de las diferencias en las estructuras económicas, es decir, por características propias del entorno en el que opera la empresa. Esta situación permite justificar la relevancia de incorporar un factor que controle esta fuente de variación en la variable dependiente determinada.

De hecho, las diferencias significativas que existen entre los sectores industriales y en la localización geográ- 
Factores explicativos de los resultados empresariales: un estudio de las empresas... Huerta, Patricia; Contreras, Sergio; Almodóvar, Paloma y Pedraja-Rejas, Liliana

\section{Tabla 6}

Resultado de técnicas de datos de panel

\begin{tabular}{|c|c|c|c|c|}
\hline \multirow[t]{2}{*}{ Variables } & \multicolumn{4}{|c|}{ Modelo de Efectos Aleatorios } \\
\hline & Estimación & Error Estándar & t-value & $\operatorname{Pr}(>|t|)$ \\
\hline Tamaño & $1.1183118^{* * *}$ & 0.0087921 & 127.1947 & $<2.2 \mathrm{e}-16$ \\
\hline Alim. y Tabaco & 0.1272690 & 0.0832944 & 1.5279 & 0.1265729 \\
\hline Bebidas & 0.8770556 *** & 0.1262148 & 6.9489 & $4.018 e-12$ \\
\hline Textil & $-0.2045908 *$ & 0.0852787 & -2.3991 & 0.0164624 \\
\hline Cuero y Calzado & -0.0892683 & 0.1133503 & -0.7875 & 0.4309907 \\
\hline Ind. De la Madera & -0.1307264 & 0.1033617 & -1.2647 & 0.2060047 \\
\hline Papel & 0.3008558 ** & 0.0983464 & 3.0591 & 0.0022282 \\
\hline Edición y Artes & $0.3292723^{* * *}$ & 0.0906303 & 3.6331 & 0.0002820 \\
\hline Prod. Químicos & $0.5607953^{* * *}$ & 0.0893142 & 6.2789 & $3.616 e-10$ \\
\hline Caucho y Plástico & 0.1925582 * & 0.0881283 & 2.1850 & 0.0289241 \\
\hline Miner. no Metál. & 0.2830422 ** & 0.0880922 & 3.2130 & 0.0013195 \\
\hline Metalúrgica & $0.4154448^{* * *}$ & 0.0947577 & 4.3843 & $1.181 \mathrm{e}-05$ \\
\hline Prod. Metálicos & $0.1888150^{*}$ & 0.0809353 & 2.3329 & 0.0196813 \\
\hline Maq. y Eq Mec. & $0.2575910^{* *}$ & 0.0853249 & 3.0189 & 0.0025458 \\
\hline Maq. De Ofc. Eq. Informáticos & 0.0174746 & 0.1225852 & 0.1426 & 0.8866494 \\
\hline Maq. Materiales elect. Electr. & 0.0953250 & 0.0886719 & 1.0750 & 0.2823984 \\
\hline Veh. de Motor & 0.1627562 & 0.0903090 & 1.8022 & 0.0715554 \\
\hline Material transporte & $0.3004214^{*}$ & 0.1198044 & 2.5076 & 0.0121782 \\
\hline Muebles & -0.0807142 & 0.0919495 & -0.8778 & 0.3800771 \\
\hline Otras Ind. Manuf. & 0.2179019 . & 0.1169254 & 1.8636 & 0.0624206 \\
\hline Aragón & 0.1457958 & 0.0872016 & 1.6719 & 0.0945814 \\
\hline Asturias & $0.2857898^{* *}$ & 0.0983090 & 2.9071 & 0.0036601 \\
\hline Baleares & -0.0825169 & 0.1393255 & -0.5923 & 0.5536961 \\
\hline Canarias & 0.2172523 & 0.1129044 & 1.9242 & 0.0543687 \\
\hline Cantabria & $0.3416166 * *$ & 0.1265029 & 2.7005 & 0.0069412 \\
\hline Castilla- la Mancha & 0.0219964 & 0.0767895 & 0.2865 & 0.7745413 \\
\hline Castilla León & $0.3193655^{* * *}$ & 0.0776125 & 4.1149 & $3.919 \mathrm{e}-05$ \\
\hline Cataluña & $0.3416107^{* * *}$ & 0.0614396 & 5.5601 & $2.797 e-08$ \\
\hline Comunidad Valenciana & 0.1535910 * & 0.0648045 & 2.3701 & 0.0178121 \\
\hline Extremadura & -0.2226930 & 0.1254985 & -1.7745 & 0.0760300 \\
\hline Galicia & 0.1357749 & 0.0817196 & 1.6615 & 0.0966638 \\
\hline Madrid & $0.2657881^{* * *}$ & 0.0622779 & 4.2678 & $2.001 \mathrm{e}-05$ \\
\hline Murcia & 0.0854746 & 0.0961543 & 0.8889 & 0.3740708 \\
\hline
\end{tabular}




\section{Tabla 6}

Resultado de técnicas de datos de panel (Continuación).

\begin{tabular}{|c|c|c|c|c|}
\hline \multirow[t]{2}{*}{ Variables } & \multicolumn{4}{|c|}{ Modelo de Efectos Aleatorios } \\
\hline & Estimación & Error Estándar & t-value & $\operatorname{Pr}(>|t|)$ \\
\hline Navarra & $0.2914063^{* *}$ & 0.0974000 & 2.9919 & 0.0027827 \\
\hline País Vasco & $0.3282056^{* * *}$ & 0.0734321 & 4.4695 & $7.965 \mathrm{e}-06$ \\
\hline La Rioja & $0.4527317^{\star \star \star}$ & 0.1298810 & 3.4857 & 0.0004938 \\
\hline Intercepto & $9.5926842 * * *$ & 0.0951328 & 100.8347 & $2.2 \mathrm{e}-16$ \\
\hline $\mathrm{N}$ & 6942 & & & \\
\hline SCT & 3817.1 & & & \\
\hline CCR & 966.64 & & & \\
\hline $\mathrm{R}^{2}$ & 74.68 & & & \\
\hline F-estadístico & $565.607^{* * *}$ & & & \\
\hline
\end{tabular}

fica se pueden deber a características propias del sector, barreras de entrada, concentración, entre otras, o bien, a características propias de la ubicación en la cual se encuentra la empresa, mayor cercanía con proveedores, mejor acceso a canales de distribución, entre otros. Lo anterior, provoca que las empresas instaladas en determinados sectores y localizaciones obtengan mejores resultados en comparación con otras organizaciones ubicadas en otros sectores o lugares geográficos.

Por último, se destaca el menor error alcanzado, un $4.4 \%$, lo cual se puede deber a diversas razones, tales como el periodo de análisis, el perfil de las empresas, la depuración de la muestra, entre otras.

\section{Conclusiones}

La preocupación sobre el estudio de los factores que provocan mejores re- sultados es un tema de interés empresarial y de amplio debate a lo largo de la literatura, que debe ser considerado por las organizaciones para observar aquellas variables que están directamente impactando en sus resultados.

Por otro lado, aunque tradicionalmente la forma en la cual las empresas miden los resultados empresariales es el ROA, también deberían observar las limitaciones que posee (Maroto, 1995; McGahan, 1999; Puig, 1996; Salgueiro, 2001) y atreverse a utilizar otras formas de medida, tales como el valor agregado, debido a su evidente contribución en el reflejo de la productividad de la empresa.

De igual modo, se ha demostrado que el efecto empresa, o los recursos propios de la empresa, es superior al efecto industria, o a las características que ésta posea sobre las empresas manufactureras. Lo anterior permite reconocer la relevancia que otorgan estos sectores a las capacidades tácitas y habilidades com- 
Factores explicativos de los resultados empresariales: un estudio de las empresas... Huerta, Patricia; Contreras, Sergio; Almodóvar, Paloma y Pedraja-Rejas, Liliana

plejas que deben desarrollar o poseer las personas que se encuentran presentes en los sectores industriales (Cuervo, 1999). Sumado a ello, resulta imprescindible resaltar la idea que aquellas empresas que deseen alcanzar mejores resultados deben considerar la ubicación geográfica o localización como un recurso estratégico que influye en su obtención de resultados.

Este trabajo avanza en la discusión del tema, introduciendo un efecto que no es considerado normalmente en los estudios tradicionales, al igual que actualiza e incorpora un horizonte temporal mayor en relación a los trabajos en esta línea. A la vez, se presenta en un solo artículo dos aspectos relevantes, explicación e influencia de los factores. Tópicos que en la mayoría de las investigaciones se analizan por separado a pesar de su estrecha vinculación. Con ello, se pretende aumentar el conocimiento sobre la importancia de considerar estos factores en la gestión empresarial, al igual que servir de orientación a otros países que compartan similitudes con las empresas de este estudio.

A través de esta investigación se confirmó la hipótesis 1 y la hipótesis 2, es decir, se descubrió que el factor tiempo, el factor localización, el factor sectorial y, principalmente, el factor empresa explican e influyen en los resultados empresariales de las empresas industriales españolas.

Por último, una limitación de este trabajo es la forma de medida de los resultados empresariales y su aplicación sólo a empresas industriales. Por lo anterior, se espera en una futura investigación utilizar una forma de medida diferente, al igual que ampliar el ámbito de aplicación a través de un análisis comparativo entre un país desarrollado y un país en vías de desarrollo. Lo anterior, tendría especial relevancia debido a que la mayoría de las investigaciones se aplican a países desarrollados, mientras que son los países en vías de desarrollo los que requieren mayor atención y estudios para potenciar su desarrollo. Con ello, podrían descubrirse cuáles son las diferencias y/o similitudes con las grandes economías y las menos desarrolladas, con la finalidad de aprender de ellas y así contribuir al debate científico sobre el tema.

\section{Referencias bibliográficas}

Amel, Dean y Froeb, Luke (1991). Do Firms Differ Much?, The Journal of Industrial Economics, Volumen 39(3), England, Sussex, England: John Wiley \& Sons Ltd., pp 323-331.

Amit, Raphael y Schoemaker, Paul (1993). "Strategic Assets And Organizational Rent", Strategic Management Journal, Volumen 14(1), England, Sussex, England: John Wiley \& Sons Ltd., pp 33-46.

Aragón, Antonio y Rubio, Alicia (2005). Factores asociados con el éxito competitivo de las Pyme industriales en España. Universia Business Review, España, Madrid: Portal Universia S.A., pp 36-49.

Ariño, Miguel; Ariño, Africa; Garcia-Castro, Roberto (2008). "A Model to Evaluate Transient Industry Effects", Managerial \& Decision Economics, Volumen 29(8), England, Sussex, England: John Wiley \& Sons Ltd., pp 629-637.

Barney, Jay (1991). "Firm Resources And Sustained Competitive Advantage", Journal Of Management, Volumen 17(1), United States, Thousand Oaks, Ca: Sage Publications Inc, pp 99-120. 
Benavente, José (2008). La Dinámica Empresarial En Chile (1999-2006). Ministerio De Economía. Gobierno De Chile. Chile.

Brush, Thomas; Bromiley, Philip y Hendrickx, Margaretha (1999). The Relative Influence of Industry and Corporation on Business Segment Performance: an Alternative Estimate, Strategic Management Journal, Volumen 20(6), England, Sussex, England: John Wiley \& Sons Ltd, pp 519-547.

Calderón, Gregorio; Álvarez, Claudia \& Naranjo, Julia (2010). "Estrategia competitiva y desempeño organizacional en empresas industriales colombianas. Innovar". Revista de Ciencias Administrativas y Sociales, Volumen 20(38), Colombia, Bogotá, Universidad Nacional de Colombia, pp 13-26.

Caloghirou, Yiannis; Protogerou, Aimilia; Spanos, Yiannis y Papagiannakis, Lefteris (2004). "Industry-Versus-Firm Specific Effects on Performance", European Management Journal, Volumen 22(2), England, Amsterdam, Netherlands: Elsevier Science Bv, Elsevier Ltd., pp 231-243.

Capar, Nejat (2009). "An Analysis of the Relationships Between International Diversification, Product Diversification, Firm Resources and Performance", The Academy Of Management Proceedings, United States, Ada, Ohio: Academy of Management, pp1-6.

Chang, Sea-Jin y Singh, Harbir (2000). “Corporate and Industry Effects on Business Unit Competitive Position", Strategic Management Journal, Volumen 21(7), England, Sussex, England: John Wiley \& Sons Ltd, pp 739-752.

Chen, Yi-Min (2009). "Determinants of Industry Performance: Region Vs. Country Effects in Knowledge-Intensive Service Industries", Service Industries Journal, London, Taylor \& Francis Group, Volumen 29(3), pp 297-316.
Claver, Enrique; Molina, José y Quer, Diego (2000). Incidencia comparada del efecto empresa y el efecto sector en la rentabilidad económica. Aplicación a una muestra de empresas de Alicante 1994-1998, Economía Industrial (334), España: Ministerio de Industria, Turismo y Comercio, pp 143-152.

Claver, Enrique; Llopis, Juan y Molina, José (2002). Recursos de la empresa y pertenencia a un sector industrial: Un estudio empírico de su influencia sobre la rentabilidad empresarial, Investigaciones Europeas de Dirección y Economía de la Empresa, Volumen 8(1), España: AEDEM, pp 39-52.

Cool, David Schendel, David y Goold, Michael (1988). "The Size, Structure, and Performance of Corporate Headquarters", Strategy Management Journal, Volumen 28(4), England, Sussex, England: John Wiley \& Sons Ltd, pp. 383-405.

Cubbin, John y Geroski, Paul (1987). "The Convergence of Profits in the Long Run: Inter-Firm and Inter-Industry Comparisons", The Journal of Industrial Economics, Volumen 35(4), England, Sussex, England: John Wiley \& Sons Ltd., pp 427-442.

Cuervo, Álvaro (1993). El papel de la empresa en la competitividad, Papeles de Economía Española (56), España: Fundación de las Cajas de Ahorro, pp 363-378.

Cuervo, Álvaro (1999). "La dirección estratégica de la empresa: reflexiones desde la economía de la empresa", Papeles de Economía Española (78-79), España: Fundación de las Cajas de Ahorro, pp. 34-55.

Díaz, María y Sánchez, Rosario (2008). Firm Size and Productivity in Spain: A Stochastic Frontier Analysis, Small Business Economics, Volumen 30(3), Netherlands: Springer, pp. 315-323. 
Factores explicativos de los resultados empresariales: un estudio de las empresas... Huerta, Patricia; Contreras, Sergio; Almodóvar, Paloma y Pedraja-Rejas, Liliana

Dunne, Timothy y Macpherson, David (1991). "A Note on Ownership And Performance in Manufacturing Firms", Souther Economic Journal, Volumen 57(4), United Status: Southern Economic Association, pp 1.164-1.169.

ESEE (1990). Encuesta sobre Estrategias Empresariales. Fundación Sociedad Estatal de Participaciones Estatales (SEPI), Madrid. http://www.funep. es/esee

Fernández, Esteban; Montes, José y Vázquez, Camilo (1996). "Efecto Industria y Conducta Empresarial”. Revista Europea de Dirección y Economía de la Empresa, Volumen 5(2), España, La Rioja: AEDEM, pp 149-158.

Fernández, Esteban; Montes, José y Vázquez, Camilo (1997). La importancia del sector como determinante del beneficio, XI Congreso Nacional de AEDEM, España: Lleida.

Fernández, Zulima (1992). "Algunas reflexiones sobre la competitividad empresarial y sus causas, información comercial española, ICE: Revista Economía (705), España: Ministerio de Industria, Turismo y Comercio, pp. 139-152.

Galán, José y Vecino, Julio (1997). “Las Fuentes de Rentabilidad de las Empresas", Revista Europea de Dirección y Economía de la Empresa, Volumen 6 (1), España: AEDEM, pp 21-36.

Galve, Carmen y Salas, Vicente (1993). "Propiedad y resultados de la gran empresa españolas", Investigaciones Económicas, Volumen 17(2), España: Fundación Empresa Pública, pp 207-238.

Garengo, Patrizia (2009). A Performance Measurement System for Smes Taking Part in Quality Award Programmes, Total Quality Management \& Business Excellence, Volumen 20(1), England, London: Taylor \& Francis Group, pp 91-105.
González, Eduardo (2000). Efecto Industria, Efecto Grupo y Efecto Empresa, $X$ Congreso Nacional de ACEDE, Oviedo.

Grant, Robert (1991). The Resource-Based Theory of Competitive Advantage. Implications for Strategy Formulation, California Management Review, Volumen 33(3), United States, Berkeley: University of California, pp 114-135.

Halkos, George y Tzeremes, Nickolaos (2007). Productivity Efficiency and Firm Size. An Empirical Analysis of Foreign Owned Companies, International Business Review, Volumen16(6), England, Amsterdam, Netherlands: Elsevier Science Bv, Elsevier Ltd., pp 713-731.

Hansen, Gary y Wernerfelt, Birger (1989). Determinants of Firm Performance: The Relative Importance of Economic and Organizational Factors, Strategic Management Journal, Volumen 10(5), England, Sussex, England: John Wiley \& Sons Ltd., pp 399-411.

Hill, Charles y Deeds, David (1996). "The Importance of Industry Structure for the Determination of Firm Profitability: A Neo-Austrian Perspective", Journal of Management Studies, Volumen 33(4), England, Sussex, England: John Wiley \& Sons Ltd., pp 429-451.

Huang, Joan y Sylvie, George (2010). "Industry and Firm Effects on Performance: Evidence from the Online News Industry in U.S.", Journal of Media Business Studies, Volumen 7(1), Sweden: Jönköping International Business School, pp 1-20.

Huerta, Patricia; Contreras, Sergio; Almodóvar, Paloma y Navas, José (2010). “Influencia del tamaño empresarial sobre los resultados: Un estudio comparativo entre empresas chilenas y españolas", Revista Venezolana de Gerencia, (50), Venezuela: Universidad Zulia, pp 207-230. 
Hull, Clyde Y Rothenberg, Sandra (2008). "Firm Performance. The Interactions of Corporate Social Performance With Innovation and Industry Differentiation", Strategy Management Journal, Volumen 29(7), England, Sussex, England: John Wiley \& Sons Ltd., pp 781-789.

Iglesias, Susana; López, Vicente y Rodríguez, Marcos (2007). "Efecto empresa versus efecto sector: Un estudio empírico en empresas gallegas y portuguesas", Revista Galega de Economía, España, Universidad Santiago de Compostela, Volumen 16(2), pp 1-13.

Kim, Mandy (2009). "The relationship between innovativeness and the performance of small and medium-size enterprises (Smes) of Malaysian Manufacturing Sector", International Journal of Management and Innovation, Volumen 1(2), United States: Word Scientific Publishing Co., pp 1-14.

Lang, Larry Y Stulz, Rene (1994). “Tobin'S Q, Corporate Diversification, and Firm Performance", Journal of Political Economy, Volumen 102(6), United States, Chicago, II: Univ Chicago Press, pp 1248-1280.

Makino, Shige; Isobe, Takehito y Chan, Christine (2004). "Does Country Matter?", Strategic Management Journal, Volumen 25(10), England, Sussex, England: John Wiley \& Sons Ltd., pp 1027-1043.

Maroto, Juan (1995). "Posibilidades del análisis económico-financiero de las empresas españolas", Papeles de Economía Española, (62), España: Fundación De Las Cajas de Ahorro, pp 113-136.

Mauri, Alfredo Y Michaels, Max (1998). "Firm and Industry Effects Within Strategic Management: An Empirical Examination", Strategic Management Journal, Volumen 19(3), England, Sussex,
England: John Wiley \& Sons Ltd., pp 211-219.

McGahan, Anita y Porter, Michael (1997). "How Much Does Industry Matter, Really?”, Strategy Management Journal, Volumen 18(S1), England, Sussex, England: John Wiley \& Sons Ltd., pp 15-30.

McGahan, Anita (1999). "The Performance of Us Corporations: 1981-1994", Journal of Industrial Economics, Volumen 47(4), England, Sussex, England: John Wiley \& Sons Ltd., pp 373-398.

Merino de Lucas, Fernando y Rodríguez, Diego (1997). "A Consistent Analysis Of Diversification Decisions With NonObservable Firm Effects", Strategic Management Journal, Volumen 18(9), England, Sussex, England: John Wiley \& Sons Ltd., pp 733-743.

Miller, Douglas (2006). "Technological diversity, related diversification, and firm performance", Strategic Management Journal, Volumen 27(7), England, Sussex, England: John Wiley \& Sons Ltd., pp 601-619.

Montgomery; Cynthia y Wernerfelt, Birger (1991). "Sources of Superior Performance: Market Share Versus Industry Effects In The U.S. Brewing Industry", Management Science, Volumen 37(8), United States, Philadelphia: Institute For Operations Research And The Management Sciences, pp 954-959.

Odagiri, Hiroyuki (1983). "Ryd Expenditures, Royalty Payments, And Sales Growth In Japanese Manufacturing Corporations". Journal of Industrial Economics, Volumen 32(1), England, Sussex, England: John Wiley \& Sons Ltd., pp 61-71.

Paliwal, Rupendra (2010). "Intra-Industry Effects of Takeovers: A Study of the Operating Performance of Rival 
Factores explicativos de los resultados empresariales: un estudio de las empresas... Huerta, Patricia; Contreras, Sergio; Almodóvar, Paloma y Pedraja-Rejas, Liliana

Firms", The International Journal Of Business And Finance Research, Volumen 4(2), United States: Institute For Business And Finance Research, pp 97-115.

Pedraja-Rejas, Liliana; Rodríguez-Ponce, Emilio y Rodríguez-Ponce, Juan (2009). "Gestión del Conocimiento, Eficacia Organizacional en Pequeñas y Medianas Empresas". Revista Venezolana de Gerencia, Volumen 14 (48), Venezuela: Universidad Zulia, pp 495-506.

Peng, Mike (2001). "The Resource-Based View and International Business", Journal of Management, Volumen 27(6), England, Amsterdam, Netherlands: Elsevier Science Bv, Elsevier Ltd., pp 803-829.

Peteraf, Margaret (1993). "The Cornerstones of Competitive Advantage. A Resource-Based View", Strategic Management Journal, Volumen 14(3), England, Sussex, England: John Wiley \& Sons Ltd., pp 179-191.

Puig, Pere (1996). La Competitivita de L'Empresa Industrial a Catalunya. Anàlisis de L'Entorn Econòmic I de las Estratègies Competitives en un Contexte de Modernització del Sector Públic. ESADE, Barcelona.

Ramírez, Marisa y Espitia, Manuel (2002). "The Impact of Product Diversification Strategy on the Corporate Performance of Large Spanish Firms", Spanish Economic Review, Volumen 4(2), Germany, Berlin: Springer, pp 119-137.

Roquebert, Jaime, Phillips, Robert Y Westfall, Peter (1996). "Markets vs. Management: What 'Drives' Profitability?", Strategic Management Journal, Volumen 17(8), England, Sussex, England: John Wiley \& Sons Ltd., pp 653-664.

Rubio, Alicia y Aragón, Antonio (2002). "Factores Explicativos del Éxito Competitivo. Un Estudio Empírico en la Pyme",
Cuadernos de Gestión, Volumen 2(1), España: Instituto de Economía Aplicada a la Empresa de la Universidad del País Vasco, pp 49-63.

Rumelt, Richard (1991). "How Much Does Industry Matter?", Strategic Management Journal, Volumen 12(3), England, Sussex, England: John Wiley \& Sons Ltd., pp167-185.

Salgueiro, Amado (2001). Indicadores de Gestión y Cuadro de Mando. Díaz de Santos, Madrid.

Schmalensee, Richard (1985). Do Markets Differ Much?, American Economic Review, Volumen 75(3), United States: American Economic Association, pp 341-351.

Scott, John y Pascoe, George (1986). Beyond Firm and Industry Effects on Profitability in Imperfect Markets, Review of Economics and Statistics, Volumen 68(2), United States: Harvard University's Kennedy School of Government, pp 284-292.

Short, Jeremy; Ketchen, David y Palmer, Timothy (2007). "Firm, Strategy Group, and Industry Influences on Performance", Strategic Management Journal, Volumen 28(2), England, Sussex, England: John Wiley \& Sons Ltd., pp 147-167.

Serrasqueiro, Zelia y Macas, Paulo (2008). "Performance And Size: Empirical Evidence From Portuguese Smes", Small Business Economic, Volumen 31(2), Netherlands: Springer, pp 195-217.

Tanriverdi, Hüseyín y Lee, Chi-Hyon (2008). "Within-Industry Diversification and Firm Performance in the Presence of Network Externalities. Evidence from the Software Industry", Academy of Management Journal, Volumen 51(2), United States, Ada, Ohio: Academy of Management, pp 381-397.

Torrents, Juan (2008). El Valor Añadido como medida de la eficacia empre- 
sarial. Tesis Doctoral, Universidad Politécnica de Catalunya, España.

Wernerfelt, Birger y Montgomery, Cynthia (1988). "Tobin'S Q And The Importance Of Focus In Firm Performance", American Economic Review, Volumen 78(1), United States, Nashville: American Economic Association, pp 246-250.

Wernerfelt, Birger (1984). "A Resource-Based View of the Firm", Strategic Manage- ment Journal, Volumen 5(2), England, Sussex, England: John Wiley \& Sons Ltd., pp 171-180.

Wiersema, Margarethe y Bowen, Harry (2008). "Corporate Diversification the Impact of Forein Competition, Industry Globalization, and Product Diversification", Strategy Management Journal, Volumen 29(2), England, Sussex, England: John Wiley \& Sons Ltd., pp 115-132. 